approximation we find that the apsidal progress per revolution is

$$
\frac{4 \pi \lambda \gamma^{2}\left(m_{1}+m_{2}\right)^{2}}{C^{2} h^{2}}
$$

The observed value requires that $\lambda$ should be nearly $3 / 2$, and if $3 / 2$ is taken, we get the result obtained by Prof. Einstein by his new specification and principles.

It may be observed that the above specification by the Lagrangean function could be generalised for any number of particles, and that it involves no departure from recognised dynamics or the normal views of space and time. It does, however, involve the conclusion that the interaction of bodies through the æther, vaguely called "gravitation," is to a very slight degree not exactly in accordance with Newton's specification. Whether such conclusion is really necessary seems still a matter of doubt.

I have not seen any discussion of the problem of two bodies on Prof. Einstein's specification, but it appears to me that an exact determination of the relative orbit when $m_{1}$ and $m_{2}$ are comparable quantities is very desirable.

Portsmouth, March 29.

\section{The Construction of a Magnetic Shell Equivalent to a Given Electric Current.}

AcCording to Amperre's theorem, the magnetic field due to an electric current flowing in any circuit is equivalent at external points to that due to a simple magnetic shell the bounding edge of which coincides with the conductor, and the strength of which is equal to the strength of the current.

It is generally understood that any surface having the circuit as its boundary will serve as the surface of an equivalent magnetic shell, and the fact that there is a restriction on the nature of the surface does not appear generally to be recognised.

Thus, for example, in Maxwell's treatise (vol. ii., p. I42) we find the following :-_"Conceive any surface $S$ bounded by the circuit and not passing through the point $\mathrm{P}$ "; while further on he says: "It is manifest that the action of the circuit is independent of the form of the surface $\mathrm{S}$, which was drawn in a perfectly arbitrary manner so as to fill it up."

I propose to show by means of a simple example that the surface is not drawn in a "perfectly arbitrary" manner.

Consider a narrow, rectangular strip of paper the opposite edges of which we shall denote by $a$ and $b$, its opposite faces by $\mathrm{A}$ and $\mathrm{B}$, and its two ends by $I$ and 2 . We shall represent the ends of the edges by $a_{1}, b_{1}, a_{2}, b_{2}$, where the suffixes refer to the corresponding ends of the strip. Now let one end of the paper be turned round through an angle $\pi$ and joined on to the other end, so that $a_{1}$ is joined to $b_{2}$ and $b_{1}$ to $a_{2}$.

Then, since $a_{1}$ is joined to $b_{2}$, the edges $a$ and $b$ form one continuous line, and, since $b_{1}^{\prime}$ is joined to $a_{2}$, this line forms a closed circuit.

Thus we may bend a wire into the form of the edge, and can imagine an electric current to flow in it.

Although the electric circuit has the form of the edge, yet we could not have a simple magnetic shell the surface of which was that of the paper.

This is easily seen, for since, in addition to the edges, the faces $\mathrm{A}$ and $\mathrm{B}$ have also become continuous one with the other, we can no longer distinguish one as positive and the other as negative. The same thing is seen if we try to imagine the surface divided up into elementary portions, in the manner conceived NO. 2633 , VOL. IO5] of by Ampère, with a current equal in strength to the given current flowing round the boundary of each.

It is easily seen that Ampère's construction fails for such a surface, which is known to mathematicians as a Möbius sheet.

Although the surface we have described would not serve as the surface of a simple magnetic shell equivalent to an electric current flowing round its boundary, yet it is possible to construct other surfaces having this boundary which would serve as surfaces of equivalent magnetic shells.

If we have one suitable surface we can obtain any number of others from it by continuous deformation while the edge remains fixed.

It is, therefore, desirable to give a general method of constructing a magnetic shell equivalent to a given electric circuit. The following appears to give a surface having the required property :-

Let $\mathrm{O}$ be a fixed point external to the circuit, and let $\mathrm{P}$ be a variable point. Let $\mathrm{P}$ travel once completely round the circuit, so that the radius vector $O P$ traces out some conical surface.

The portion of this conical surface containing $O$ and bounded by the circuit might then be taken as the surface of the equivalent magnetic shell.

In the particular case of the circuit we have considered (as well as in many others) the surface will cut itself, but will, nevertheless, have two distinct faces, one of which may be taken as positive and the other as negative. It thus appears to satisfy the necessary conditions.

March 30.

\section{Volcanic Rocks in the Anglo-Egyptian Sudan.}

IN connection with Prof. J. W. Gregory's reference in NATURE of February 19, p. 667 , to the discovery of the Bayuda volcanic field, and Mr. Campbell Smith's record of a riebeckite-rhyolite which constituted a number of stone implements found at Jebel Katul, in Northern Kordofan (ibid., February 26 , p. 693), some further notes may be of interest.

The rock collected by Sir Herbert Jackson at Merowe is a basaltic scoria, and the specimens either float or just sink in water. A few crystals of olivine are visible to the eve, and, urder the microscope, a regular basaltic ground-mass, including felspar, iron ores, and probablv glass, can be recognised in the powdered rock. The specimens have evidently been transported by a stream system which drains from the south-east and debouches on the river at the spot where they were found. Save for the neighbourhoods of the river and a few routes by which travellers avoid the long journey around the Abu Hamed bend of the Nile, the maps of the Bayuda Desert are almost blank. Near one of the routes a surveyor has recorded "Hosh Eddalam, crater," and the name means a dark enclosure. Some of the older travellers mistook ironstone concretions for volcanic bombs, and as the surfaces of many rocks are darkened in the desert such a record of a crater did not call for particular note until evidence of extrusive rocks appeared. It is situated in latitude $\mathrm{I}^{\circ} 20^{\prime} \mathrm{N}$, , longitude $32^{\circ} 3 \mathrm{I}^{\prime} \mathrm{E}$., and consequently lies to the west of the route taken by Dr. Chalmers Mitchell. The volcanic field seen from the air probably does not lie on the established routes, as it would certainly have been referred to in reports, even if it were not described. Presumably there can be no doubt about the existence of craters seen by an observer such as Dr. Chalmers Mitchell, but the results of an examination on the ground will be of interest, even if onlv to know the tvpes of rocks involved.

Mr. Stanlev C. Dunn records the presence of rhyolites and felsites near Jakdul, and these are doubtless 